\title{
7-Dimensional Compact Riemannian Manifolds with Killing Spinors
}

\author{
Thomas Friedrich and Ines Kath \\ Sektion Mathematik, Humboldt Universität, Postfach 1297, 1086 Berlin, \\ Federal Republic of Germany
}

Received February 27, 1989

\begin{abstract}
Using a link between Einstein-Sasakian structures and Killing spinors we prove a general construction principle of odd-dimensional Riemannian manifolds with real Killing spinors. In dimension $n=7$ we classify all compact Riemannian manifolds with two or three Killing spinors. Finally we classify nonflat 7-dimensional Riemannian manifolds with parallel spinor fields.
\end{abstract}

\section{Introduction}

A Killing spinor on a Riemannian spin manifold $\left(M^{n}, g\right)$ is a section $\psi$ of the spin bundle $S$ satisfying, for any vector field $X$, the differential equation

$$
\nabla_{X} \psi=\lambda X \psi,
$$

where $\lambda \neq 0$ is a constant and $X \psi$ denotes the Clifford multiplication of the vector $X$ by the spinor $\psi$. Solutions of this equation occur quite naturally in Differential Geometry as well as in Mathematical Physics. For example, on a compact Riemannian spin manifold with non-negative scalar curvature $R$ there is a lower bound involving $R$ for the first eigenvalue of the Dirac operator, and eigenspinors to this lower bound are Killing spinors (see [8, 19, 30]). Furthermore, Killing spinors are special solutions of the so-called twistor equation and in case of a compact manifold they generate - up to a conformal change of the metric - all solutions of the twistor equation (see [31]).

The construction of models in supergravity depends on Riemannian manifolds with Killing spinors. There are several papers (see for example [6,32]) investigating, from this point of view, the properties of Riemannian manifolds with Killing spinors as well as containing the construction of examples.

The existence of a Killing spinor imposes algebraic conditions on the Weyl tensor of the space (see [9]) and on the covariant derivative of the curvature tensor; in particular $M^{n}$ must be an Einstein space (see [8]). The constant $\lambda$ is given by

$$
\lambda^{2}=\frac{1}{4} \frac{R}{n(n-1)} .
$$


Consequently there are two possibilities. In case $\lambda$ is an imaginary number, $M^{n}$ is a complete, non-compact Einstein space with an imaginary Killing spinor. Recently, Helga Baum classified all such Einstein spaces (see [2 and 3]). In case $\lambda$ is a real number, $M^{n}$ is a compact Einstein space with positive scalar curvature. In the dimensions 5, 6, and 7 we know many examples of compact Einstein spaces with real Killing spinors (see $[6,8,11,12,32])$. Moreover, in dimension five there is a one-to-one correspondence between real Killing spinors and Einstein-Sasakian structures on a simply connected manifold (see [12]). This basic observation yields classification results concerning 5-dimensional compact Riemannian manifolds with Killing spinors (see [12]).

In this paper we first of all prove that any odd-dimensional simply-connected Einstein-Sasakian manifold admits at least two Killing spinors. In particular, starting with a Kähler-Einstein space $X^{2 n}$ of positive scalar curvature and taking into consideration a suitable $S^{1}$-fibre bundle $M^{2 n+1}$ over $X^{2 n}$, it is not hard to see that $M^{2 n+1}$ has an Einstein-Sasakian structure. Consequently we obtain a construction of odd-dimensional compact Einstein manifolds with two real Killing spinors depending on a Kähler-Einstein metric. This general construction principle includes all known examples of compact Riemannian manifolds with at least two real Killing spinors (see $[6,8,12,32]$ ) and indicates many new Einstein spaces like this, since recently Koiso, Sakane, Tian, and Yau (see [27, 35, 36]) proved the existence of Kähler-Einstein metrics on several compact manifolds with positive first Chern class.

The second main emphasis of this paper is the classification of 7-dimensional compact manifolds with two or three real Killing spinors. Conversely to our general construction principle we prove that, especially in dimension seven, any pair of real Killing spinors defines an Einstein-Sasakian structure on the underlying space. Under some regularity assumption-this observation yields a description of all compact Riemannian manifolds with two real Killing spinors. Applying twistor theory and, in particular, the classification of all compact Kählerian twistor spaces (see [15,21]), we classify all compact Riemannian manifolds with at least three real Killing spinors.

Finally we explain that the same techniques can also be applied in order to classify non-flat Riemannian manifolds with parallel spinor fields.

\section{Killing Spinors and Einstein-Sasakian Structures}

Let $\left(M^{n}, g\right)$ be a compact connected Riemannian spin manifold with scalar curvature $R$. Denote by $Q$ the principal $S O(n)$-bundle and fix a spin structure, i.e. a principal Spin $(n)$-bundle $P$ and a covering $P \rightarrow Q$. The spinor bundle of $M^{n}$ is the associated bundle

$$
S=P \times_{\operatorname{Spin}(n)} \Delta_{n},
$$

where $\chi: \operatorname{Spin}(n) \rightarrow G L\left(\Delta_{n}\right)$ is the complex $2^{\left[\frac{n}{2}\right]}$-dimensional spin representation. A real Killing spinor on $M^{n}$ is a section $\psi$ of the spin bundle satisfying the following first order partial differential equation:

$$
\nabla_{X} \psi=\lambda X \psi,
$$

where $X \psi$ denotes the Clifford multiplication of the vector $X$ by the spinor $\psi$, and $\lambda \neq 0$ is a real number. The inner product $\left(\psi_{1}, \psi_{2}\right)$ of two real Killing spinors is 
constant on $M^{n}$. A manifold with a non-trivial Killing spinor must be an Einstein space with scalar curvature $R=4 n(n-1) \lambda^{2}$ (see [8]). The existence of a Killing spinor imposes an algebraic condition on the Weyl tensor $W: \Lambda^{2}\left(M^{n}\right) \rightarrow \Lambda^{2}\left(M^{n}\right)$ of the manifold, namely

$$
W\left(\eta^{2}\right) \psi=0
$$

for any 2-form $\eta^{2} \in \Lambda^{2}\left(M^{n}\right)$ (see [9]).

We introduce now some notations concerning contact structures. A general reference is $[5,23]$. A contact metric structure on an odd-dimensional manifold $M^{2 k+1}$ consists of a 1 -form $\eta$, a vector field $\xi$, a $(1,1)$-tensor $\varphi$ and a Riemannian metric $g$ such that the following conditions are satisfied:

a) $\eta \wedge(d \eta)^{k} \neq 0$

b) $\eta(\xi)=1, \varphi(\xi)=0$,

c) $\varphi^{2}=-\mathrm{Id}+\eta \otimes \xi$

d) $g(\varphi(X), \varphi(Y))=g(X, Y)-\eta(X) \eta(Y)$,

e) $d \eta(X, Y)=2 g(X, \varphi(Y))$, where we use the formula $d \eta(X, Y)=X \eta(Y)-Y \eta(X)$ $-\eta([X, Y])$ for the exterior derivative of a 1 -form $\eta$.

In particular we have $\eta(X)=g(X, \xi)$.

In case $\xi$ is a Killing vector field, we call the given structure on $M^{2 k+1}$ a $K$-contact structure. This is equivalent to

$$
\nabla_{X} \xi=-\varphi(X)
$$

and then

$$
\mathscr{L}_{\xi} g=0, \quad \mathscr{L}_{\xi} \eta=0, \quad \mathscr{L}_{\xi} \varphi=0
$$

hold.

A Sasakian manifold is a $K$-contact structure satisfying the integrability condition

$$
[\varphi, \varphi]+d \eta \xi=0
$$

or, equivalently,

g)

$$
\left(\nabla_{X} \varphi\right)(Y)=g(X, Y) \xi-\eta(Y) X .
$$

The curvature tensor $\mathscr{R}$ and the Ricci tensor Ric of a Sasakian manifold commute with $\varphi$ and have the following properties:

$$
\begin{gathered}
\mathscr{R}(X, Y) \xi=\eta(Y) X-\eta(X) Y, \\
\operatorname{Ric}(X, \varphi(Y))=\sum_{\alpha=1}^{2 k+1} \mathscr{R}\left(X, X_{\alpha}, Y, \varphi\left(X_{\alpha}\right)\right)+(1-2 k) g(\varphi(X), Y),
\end{gathered}
$$

where $X_{1}, \ldots, X_{2 k+1}$ is an orthonormal frame with respect to the metric $g$. In particular, if $M^{2 k+1}$ is an Einstein-Sasakian manifold, then its scalar curvature equals $R=2 k(2 k+1)$ and we have

$$
2 g(X, \varphi(Y))=\sum_{\alpha=1}^{2 k+1} \mathscr{R}\left(X, Y, X_{\alpha}, \varphi\left(X_{\alpha}\right)\right)
$$

Example 1 (see [26]). Consider a Kähler-Einstein manifold $\left(X^{2 m}, \bar{g}, \bar{J}\right)$ with scalar curvature $\bar{R}=4 m(m+1)$. 
Let $A$ be the maximal integer such that $\frac{1}{A} c_{1}\left(X^{2 m}\right):=c_{1}^{*}$ is an integral cohomology class. Then there exists a principal $S^{1}$ bundle $\pi: M^{2 m+1} \rightarrow X^{2 m}$ defined by $c_{1}^{*}$ as well as a connection form $\eta$ with

$$
d \eta=\frac{2(m+1)}{A} \bar{\Omega},
$$

where $\bar{\Omega}$ denotes the Kähler form of $X^{2 m}$.

The manifold $M^{2 m+1}$ is simply connected and admits a spin structure. Using the Kähler-Einstein metric $\bar{g}$ on $X^{2 m}$ and the connection $\eta$ we obtain a unique Einstein metric $g$ on $M^{2 m+1}$. Then $M^{2 m+1}$ is an Einstein-Sasakian manifold.

Proof. The Kähler-Einstein manifold $X^{2 m}$ is simply connected (see [25]) and therefore the exact sequence

$$
\rightarrow \pi_{2}\left(X^{2 m}\right) \stackrel{\partial}{\longrightarrow} \pi_{1}\left(S^{1}\right) \rightarrow \pi_{1}\left(M^{2 m+1}\right) \rightarrow \pi_{1}\left(X^{2 m}\right)=0
$$

yields that $\pi_{1}\left(M^{2 m+1}\right)$ is trivial or a cyclic group. In particular, we conclude

$$
\pi_{1}\left(M^{2 m+1}\right)=H_{1}\left(M^{2 m+1} ; Z\right) \text {. }
$$

Using the Thom-Gysin sequence of the $S^{1}$-bundle and the Poincare duality, we obtain

$$
H_{1}\left(M^{2 m+1} ; Z\right)=H^{2 m}\left(M^{2 m+1} ; Z\right)=H^{2 m}\left(X^{2 m} ; Z\right) / c_{1}^{*} \cdot H^{2 m-2}\left(X^{2 m} ; Z\right) .
$$

Since $c_{1}^{*}$ is not a multiple of an integral cohomology class, it turns out that the homomorphism

$$
c_{1}^{*} \cup: H^{2 m-2}\left(X^{2 m} ; Z\right) \rightarrow H^{2 m}\left(X^{2 m} ; Z\right)
$$

is surjective. Finally we obtain $\pi_{1}\left(M^{2 m+1}\right)=0$.

The second Stiefel-Whitney classes are related by

$$
\omega_{2}\left(M^{2 m+1}\right)=\pi^{*} \omega_{2}\left(X^{2 m}\right) \text {. }
$$

In case $X^{2 m}$ admits a spin structure, $M^{2 m+1}$ has a spin structure, too. Consider now the case

$$
0 \neq \omega_{2}\left(X^{2 m}\right) \equiv c_{1}\left(X^{2 m}\right) \bmod 2 \text {. }
$$

Then

$$
c_{1}^{*} \equiv c_{1}\left(X^{2 m}\right) \bmod 2,
$$

and from the exactness of the Thom-Gysin sequence with $Z_{2}$-coefficients we obtain

$$
\omega_{2}\left(M^{2 m+1}\right)=\pi^{*} \omega_{2}\left(X^{2 m}\right) \equiv \pi^{*} c_{1}\left(X^{2 m}\right) \equiv \pi^{*} c_{1}^{*}=0 .
$$

By the classical Weyl theorem (see [28]) there exists a connection form $\eta$ on the principal $S^{1}$-bundle $\pi: M^{2 m+1} \rightarrow X^{2 m}$ such that

$$
d \eta=2 \pi c_{1}^{*}=\frac{2 \pi}{A} c_{1}=\frac{1}{A} \bar{g}(\overline{\operatorname{Ric}}(\cdot), \cdot)=\frac{2(m+1)}{\mathrm{A}} \bar{\Omega} .
$$

This connection splits the tangent bundle of $M^{2 m+1}$ into the vertical and a horizontal part,

$$
T M^{2 m+1}=T^{v} \oplus T^{h}
$$


We define a metric $g$ on $M^{2 m+1}$ by pulling back the metric $\bar{g}$ to the horizontal subspaces and by adding the $r$-fold of the metric of the fibre $S^{1}$. Then there is precisely one parameter $r_{0}$ such that $g$ becomes an Einstein metric. The 1-form $\eta$ gives us now a $K$-contact structure and the Sasakian integrability condition is satisfied since $X^{2 m}$ is a complex manifold (see [5]).

Theorem 1. Let $\left(M^{n}, \varphi, \xi, \eta, g\right)$ be a simply connected Einstein-Sasakian manifold with spin structure.

a) If $n \equiv 1 \bmod 4$, then $M^{n}$ admits at least one Killing spinor for each of the values $\lambda= \pm \frac{1}{2}$

b) If $n \equiv 3 \bmod 4$, then $M^{n}$ admits at least two Killing spinors for one of these values $\lambda= \pm \frac{1}{2}$.

Proof. We define two subbundles $E_{ \pm}$of the spinor bundle $S$ by

$$
E_{ \pm}=\{\psi \in S:( \pm 2 \varphi(X)+\xi X-X \xi) \psi=0\} .
$$

Furthermore, we introduce the covariant derivatives

$$
\nabla_{X}^{ \pm} \psi:=\nabla_{X} \psi \pm \frac{1}{2} X \psi
$$

First of all we observe that $\nabla^{ \pm}$are connections in $E_{ \pm}$. We differentiate the equation $( \pm 2 \varphi(X)+\xi X-X \xi) \psi=0$ with respect to $Y$ :

$$
\left\{ \pm 2\left(\nabla_{Y} \varphi\right)(X)-\varphi(Y) X+X \varphi(Y)\right\} \psi+\{ \pm 2 \varphi(X)+\xi X-X \xi\} \nabla_{Y} \psi=0
$$

This equation is equivalent to

$$
\begin{aligned}
& \left\{ \pm 2\left(\nabla_{Y} \varphi\right)(X)-\varphi(Y) X+X \varphi(Y)-\varphi(X) Y \mp \frac{1}{2} \xi X Y \pm \frac{1}{2} X \xi Y\right\} \psi \\
& \quad+\{ \pm 2 \varphi(X)+\xi X-X \xi\} \nabla_{Y}^{ \pm} \psi=0,
\end{aligned}
$$

and we have to show that the first term of the last equation vanishes. A direct calculation yields this result using the properties of the Sasakian manifold and the equation defining the bundle $E_{ \pm}$:

$$
\begin{aligned}
\{ \pm & \left.2\left(\nabla_{\gamma} \varphi\right)(X)-\varphi(Y) X+X \varphi(Y)-\varphi(X) Y \mp \frac{1}{2} \xi X Y+\frac{1}{2} X \xi Y\right\} \psi \\
= & \left\{ \pm 2 g(X, Y) \xi \mp 2 \eta(X) Y-\varphi(Y) X+X \varphi(Y)-\varphi(X) Y \mp \frac{1}{2} \xi X Y \pm \frac{1}{2} X \xi Y\right\} \psi \\
= & \left\{ \pm 2 g(X, Y) \xi \mp 2 \eta(X) Y+2 X \varphi(Y)+Y \varphi(X) \mp \frac{1}{2} \xi X Y \pm \frac{1}{2} X \xi Y\right\} \psi \\
= & \left\{ \pm 2 g(X, Y) \xi \mp 2 \eta(X) Y \mp X \xi Y \pm X Y \xi \mp \frac{1}{2} Y \xi X \pm \frac{1}{2} Y X \xi \mp \frac{1}{2} \xi X Y \pm \frac{1}{2} X \xi Y\right\} \psi \\
= & \left\{ \pm 2 g(X, Y) \xi \mp 2 \eta(X) Y \mp \frac{1}{2} X \xi Y+X Y \xi \pm g(\xi, X) Y \pm \frac{1}{2} Y X \xi \pm \frac{1}{2} Y X \xi\right. \\
& \left. \pm g(X, \xi) Y \pm \frac{1}{2} X \xi Y\right\} \psi=0 .
\end{aligned}
$$

The curvature $R^{ \pm}$of the connection $\nabla^{ \pm}$is given by

$$
R^{ \pm}(X, Y) \psi=\mathscr{R}(X, Y) \psi+\frac{1}{4}(X Y-Y X) \psi .
$$

Now we prove that $R^{ \pm}$vanishes on $E_{ \pm}$, i.e. $\left(E_{ \pm}, \nabla^{ \pm}\right)$are flat bundles. Fix locally an orthonormal frame

$$
X_{1}, X_{2}=\varphi\left(X_{1}\right), \ldots, X_{n-2}, X_{n-1}=\varphi\left(X_{n-2}\right), \xi .
$$

If $\psi \in E_{ \pm}$, we have

$$
X_{i} \varphi\left(X_{i}\right) \psi=\mp \xi \psi
$$

and $\mp \xi X_{i} \varphi\left(X_{j}\right) \psi=X_{i} X_{j} \psi$. 
Suppose $X_{i} \neq X_{j}, \varphi\left(X_{j}\right)$ and consider two spinors $\psi_{1}, \psi_{2} \in E_{ \pm}$. Then we obtain

$$
\begin{aligned}
& \left(X_{i} X_{j} \psi_{1}, \psi_{2}\right)=\mp\left(\xi X_{i} \varphi\left(X_{j}\right) \psi_{1}, \psi_{2}\right) \\
& \quad= \pm\left(\psi_{1}, \varphi\left(X_{j}\right) X_{i} \xi \psi_{2}\right)= \pm\left(\psi_{1}, \xi \varphi\left(X_{j}\right) X_{i} \psi_{2}\right) \\
& \quad=\mp\left(\psi_{1}, \xi X_{i} \varphi\left(X_{j}\right) \psi_{2}\right)=\left(\psi_{1}, X_{i} X_{j} \psi_{2}\right) \\
& \quad=\left(X_{j} X_{i} \psi_{1}, \psi_{2}\right)=-\left(X_{i} X_{j} \psi_{1}, \psi_{2}\right) .
\end{aligned}
$$

Hence, in case $X_{i} \neq X_{j}, \varphi\left(X_{j}\right)$, the spinor $X_{i} X_{j} \psi$ is orthogonal to $E_{ \pm}$. Therefore the $E_{ \pm}$-part of

$$
\mathscr{R}(X, Y) \psi=\frac{1}{4} \sum_{i, j} \mathscr{R}\left(X, Y, X_{i}, X_{j}\right) X_{i} X_{j} \psi
$$

is given by

$$
\pm \frac{1}{2} \sum_{\alpha=1}^{k} \mathscr{R}\left(X, Y, X_{2 \alpha-1}, \varphi\left(X_{2 \alpha-1}\right)\right) \xi \psi= \pm \frac{1}{2} g(X, \varphi(Y)) \xi \psi
$$

where the last equality results from the above mentioned properties of the curvature tensor in an Einstein-Sasakian space. This is precisely the $E_{ \pm}$-part of $\frac{1}{4}(Y X-X Y) \psi$ for $\psi \in E_{ \pm}$. It remains to calculate the dimension of the bundle $E_{ \pm}$. This is a purely algebraic question. We consider the spin module $\Delta_{n}$ and the subspaces

$$
E_{ \pm}=\left\{\psi \in \Delta_{n}:\left( \pm X_{2 \alpha}+\xi X_{2 \alpha-1}\right) \psi=0 \text { for } \alpha=1, \ldots, k\right\}
$$

$(n=2 k+1)$. Using a realization of the Clifford algebra one checks that

$$
\begin{gathered}
\operatorname{dim} E_{+}=\operatorname{dim} E_{-}=1 \text { in case } n \equiv 1 \bmod 4, \\
\operatorname{dim} E_{+}=2, \operatorname{dim} E_{-}=0 \text { in case } n \equiv 3 \bmod 4 .
\end{gathered}
$$

Remark. Theorem 1 together with Example 1 yields a construction of odddimensional compact Einstein spaces with at least two Killing spinors. For any Kähler-Einstein manifold $X^{2 m}$ with positive scalar curvature we consider the $S^{1}$-bundle $M^{2 m+1}$ over $X^{2 m}$ with the Chern class $c_{1}^{*}=\frac{1}{A} c_{1}\left(X^{2 m}\right)$. Then $M^{2 m+1}$ admits an Einstein metric with two Killing spinors. Recently many new KählerEinstein metrics with positive scalar curvature have been constructed (see [17, 27, $35,36]$ ). Let us apply this construction to the Kähler manifolds $X^{6}=C P^{3}, F(1,2)$, $S^{2} \times S^{2} \times S^{2}, S^{2} \times C P^{2}$ or to the Graßmann manifold $G_{5,2}$. Then we obtain the 7-dimensional Einstein manifolds $M^{7}=S^{7}, S U(3) / S^{1}=N(1,1)$,

$$
\begin{gathered}
Q(1,1,1)=[S U(2) \times S U(2) \times S U(2)] / U(1) \times U(1), \\
M(3,2)=[S U(3) \times S U(2) \times U(1)] / S U(2) \times U(1) \times U(1)
\end{gathered}
$$

and an Einstein metric on the Stiefel manifold $V_{5,2}$, respectively, with two Killing spinors. These examples of Riemannian manifolds with two Killing spinors are well-known (see [6 and 8]). Moreover, let $\mathscr{F}_{k}(3 \leqq k \leqq 8)$ be one of the del Pezzosurfaces with a Kähler-Einstein metric (see [36]). Then our construction yields for $X^{4}=\mathscr{F}_{k}$ and $X^{6}=S^{2} \times \mathscr{F}_{k}$ a family of Riemannian metrics on the corresponding 5and 7-dimensional manifolds with two Killing spinors. 


\section{Some Special Properties of the Seven-Dimensional Spin Representation}

The seven-dimensional spin representation $\Delta_{7}$ is a real representation since the real Clifford algebra Cliff(7) is isomorphic to $M_{R}(8) \oplus M_{R}(8)$. In all calculations we use the following realization of this real spin representation, which we also denote by $\Delta_{7}$ :

$$
\begin{aligned}
& X_{1}=-E_{12}+E_{34}+E_{56}-E_{78}, \\
& X_{2}=E_{18}+E_{27}-E_{36}-E_{45}, \\
& X_{3}=-E_{17}+E_{28}+E_{35}-E_{46}, \\
& X_{4}=-E_{13}-E_{24}+E_{57}+E_{68}, \\
& X_{5}=E_{14}-E_{23}-E_{58}+E_{67}, \\
& X_{6}=-E_{16}+E_{25}-E_{38}+E_{47}, \\
& X_{7}=-E_{15}-E_{26}-E_{37}-E_{48},
\end{aligned}
$$

where $E_{i j}$ is the standard basis of the Lie algebra $s o(8)$ :

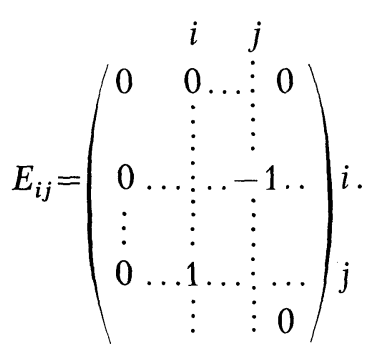

Denote by $u_{i}(1 \leqq i \leqq 8)$ the standard basis of $\Delta_{7} \approx R^{8}$. Given $u_{1}, u_{2}, \ldots, u_{k}$ we consider the isotropy group

$$
H\left(u_{1}, \ldots, u_{k}\right) \subset \operatorname{Spin}(7) H\left(u_{1}, \ldots, u_{k}\right)=\left\{g \in \operatorname{Spin}(7): g u_{\alpha}=u_{\alpha}, 1 \leqq \alpha \leqq k\right\}
$$

and its Lie algebra $h\left(u_{1}, \ldots, u_{k}\right)$. It is well-known (see [33]) that the Lie algebras $h\left(u_{1}\right), h\left(u_{1}, u_{2}\right), h\left(u_{1}, u_{2}, u_{3}\right)$ are isomorphic to the Lie algebras $g_{2}, \underline{s u}(3), \underline{s u}(2)$, respectively.

Precisely we have

Proposition 1. The Lie algebra $h\left(u_{1}, u_{2}, u_{3}\right)$ is isomorphic to su(2) and, represented as a subalgebra of the Clifford algebra, given by the equations

$$
h\left(u_{1}, u_{2}, u_{3}\right)=\left\{\begin{array}{c}
\sum_{i<j} w_{i j} X_{i} X_{j}: w_{1 i}=w_{4 i}=w_{5 i}=0 \text { for } 1 \leqq i \leqq 7 \\
w_{23}+w_{67}=0, w_{37}=w_{26}, w_{27}+w_{36}=0
\end{array}\right\}
$$

The dimensions of the Stiefel manifolds $V_{k}\left(\Delta_{7}\right)=V_{k}\left(R^{8}\right)$ are given by

$$
\begin{gathered}
\operatorname{dim} V_{1}\left(\Delta_{7}\right)=7=\operatorname{dim} \operatorname{Spin}(7)-\operatorname{dim} H\left(u_{1}\right), \\
\operatorname{dim} V_{2}\left(\Delta_{7}\right)=13=\operatorname{dim} \operatorname{Spin}(7)-\operatorname{dim} H\left(u_{1}, u_{2}\right), \\
\operatorname{dim} V_{3}\left(\Delta_{7}\right)=18=\operatorname{dim} \operatorname{Spin}(7)-\operatorname{dim} H\left(u_{1}, u_{2}, u_{3}\right) .
\end{gathered}
$$


Hence, Spin(7) acts transitively on $V_{1}\left(\Delta_{7}\right), V_{2}\left(\Delta_{7}\right)$ and on $V_{3}\left(\Delta_{7}\right)$. With respect to $\pi_{1}\left(V_{k}\left(\Delta_{7}\right)\right)=\pi_{2}\left(V_{k}\left(\Delta_{7}\right)\right)=0$ for $k=1,2,3$ (see [22]) the isotropy groups $H\left(u_{1}\right)$, $H\left(u_{1}, u_{2}\right)$, and $H\left(u_{1}, u_{2}, u_{3}\right)$ are simply connected, i.e. isomorphic to

$$
\begin{gathered}
H\left(u_{1}\right)=G_{2}, \\
H\left(u_{1}, u_{2}\right)=S U(3), \\
H\left(u_{1}, u_{2}, u_{3}\right)=S U(2) .
\end{gathered}
$$

Consider now a seven-dimensional Riemannian manifold and the real spinor bundle $S$ as well as the orthonormal sections $\psi_{1}, \ldots, \psi_{k}(1 \leqq k \leqq 3)$ in $S$. Then we obtain a $G_{2^{-}}, S U(3)-, S U(2)$-reduction $Q\left(\psi_{1}\right), Q\left(\psi_{1}, \psi_{2}\right), Q\left(\psi_{1}, \psi_{2}, \psi_{3}\right)$ of the principal Spin(7)-bundle $P$ and the frame bundle $Q$. For example, given three orthogonal spinors $\psi_{1}, \psi_{2}, \psi_{3}$ as equivariant maps $\psi_{a}: P \rightarrow \Delta_{7}(\alpha=1,2,3)$ we define

$$
Q\left(\psi_{1}, \psi_{2}, \psi_{3}\right)=\left\{p \in P: \psi_{\alpha}(p)=u_{\alpha}, \alpha=1,2,3\right\} .
$$

\section{Estimates for the Maximal Number of Independent Killing Spinors on 7-Manifolds}

Since the complex spin representation in dimension seven is the complexification of the real representation described above, the space of all real Killing spinors in the complex bundle is the complexification of the space of all Killing spinors in the real spinor bundle. Therefore we consider only real Killing spinors in the real spinor bundle. For a compact Einstein manifold $M^{7}$ with scalar curvature $R>0$ we denote by $m_{ \pm}$the dimension of the space of all positive (negative) Killing spinors:

$$
m_{ \pm}=\operatorname{dim}\left\{\psi \in \Gamma(S): \nabla_{X} \psi= \pm \frac{1}{2} \sqrt{\frac{R}{42}} X \cdot \psi\right\} .
$$

Theorem 2 (see [32 or 18]). If $m_{+}>0$ and $m_{-}>0$, then $M^{7}$ is isometric to the sphere $S^{7}$.

Proof. Given two Killing spinors $\psi_{+}, \psi_{-}$satisfying the equations

$$
\nabla_{X} \psi_{ \pm}= \pm \frac{1}{2} \sqrt{\frac{R}{42}} X \cdot \psi_{ \pm}
$$

we consider the function $f=\left(\psi_{+}, \psi_{-}\right)$. An obvious calculation yields

$$
\Delta f=\frac{R}{6} f .
$$

In case $f \neq 0, M^{7}$ must be isometric to the sphere $S^{7}$ by the Obata's theorem (see [4]).

In case $f \equiv 0$, i.e. $\psi_{+}$and $\psi_{-}$are orthogonal, we consider the 1 -form defined by

$$
\omega(X)=\left(X \psi_{+}, \psi_{-}\right) .
$$

With respect to

$$
\operatorname{dim}\left\{\eta \in S:\left(\eta, \psi_{-}\right)=0\right\}=7=\operatorname{dim} T M^{7}
$$


the 1-form $\omega$ vanishes nowhere. On the other hand, $\omega$ is a parallel form:

$$
\begin{aligned}
\left(\nabla_{X} \omega\right)(Y) & =X\left(Y \psi_{+}, \psi_{-}\right)-\left(\left(\nabla_{X} Y\right) \psi_{+}, \psi_{-}\right) \\
& =\left(Y \nabla_{x} \psi_{+}, \psi_{-}\right)+\left(Y \psi_{+}, \nabla_{x} \psi_{-}\right) \\
& =\frac{1}{2} \sqrt{\frac{R}{42}}\left\{\left(X Y \psi_{+}, \psi_{-}\right)+\left(Y X \psi_{+}, \psi_{-}\right)\right\} \\
& =-\sqrt{\frac{R}{42}} g(X, Y)\left(\psi_{+}, \psi_{-}\right)=-\sqrt{\frac{R}{42}} g(X, Y) \cdot f, \\
& =0
\end{aligned}
$$

since we have $f \equiv 0$ in the situation considered.

Now the Weitzenböck formula for 1-forms yields

$$
R \cdot \omega=0,
$$

i.e. $\omega=0$, a contradiction.

Theorem 3. Let $\left(M^{7}, g\right)$ be a compact connected Riemannian spin manifold. If $m_{+}>3$ or $m_{-}>3$, then $M^{7}$ is a space of constant sectional curvature.

Proof. We consider again the real spinor representation $\Delta_{7} \approx R^{8}$ and remark that the isotropy group $H\left(u_{1}, u_{2}, u_{3}\right)$ acts trivially on $u_{4}$ and coincides with the usual $S U(2)$-action on

$$
\operatorname{Lin}_{R}\left(u_{5}, u_{6}, u_{7}, u_{5}\right)=R^{4}=\mathbb{C}^{2} \text {. }
$$

Suppose now that $M^{7}$ admits four Killing spinors $\psi_{1}, \ldots, \psi_{4}$ with $\left(\psi_{i}, \psi_{j}\right)=\delta_{i j}$. In the reduction $Q\left(\psi_{1}, \psi_{2}, \psi_{3}\right)$ of the frame bundle we choose a section $X=\left(X_{1}, \ldots, X_{7}\right)$ such that $\psi_{4}=\lambda_{4} u_{4}+\lambda_{5} u_{5}$ holds. Suppose $\lambda_{5} \equiv 0$ and denote by $w_{i j}$ the connection forms of the Levi-Civita connection with respect to this frame on an open set $U \subset M^{7}$. The equations

$$
\nabla_{X_{2}} \psi_{\alpha}=\frac{1}{2} \sqrt{\frac{R}{42}} X_{2} \cdot \psi_{\alpha} \quad(1 \leqq \alpha \leqq 4)
$$

provide the conditions

$$
\begin{gathered}
w_{13}\left(X_{2}\right)-w_{46}\left(X_{2}\right)-w_{57}\left(X_{2}\right)=-\sqrt{\frac{R}{42}}, \\
w_{13}\left(X_{2}\right)-w_{46}\left(X_{2}\right)+w_{57}\left(X_{2}\right)=\sqrt{\frac{R}{42}}, \\
w_{13}\left(X_{2}\right)+w_{46}\left(X_{2}\right)-w_{57}\left(X_{2}\right)=\sqrt{\frac{R}{42}}, \\
w_{13}\left(X_{2}\right)+w_{46}\left(X_{2}\right)+w_{57}\left(X_{2}\right)=-\sqrt{\frac{R}{42}},
\end{gathered}
$$

a contradiction. In case $\lambda_{5} \neq 0$ at a point $m \in M^{7}$, we know that the Weyl tensor $W$ has to satisfy the algebraic equations

$$
W\left(\eta^{2}\right) \cdot \psi_{\alpha}=0
$$


for $\alpha=1, \ldots, 4$ and any 2 -form $\eta^{2}$. Using the equations describing the Lie algebra $\underline{h}\left(u_{1}, u_{2}, u_{3}\right)$ after some obvious calculations we obtain that the Weyl tensor $W$ vanishes at the point $p$. Consequently $W$ vanishes on an open and dense set, i.e. $M^{7}$ is a conformally flat Einstein space.

\section{7-Manifolds with Two Independent Killing Spinors}

In dimension seven we prove now the inversion of Theorem 1. Given a sevendimensional Einstein manifold $M^{7}$ with scalar curvature $R=42$ and two independent Killing spinors $\psi_{1}, \psi_{2}$. Without loss of generality we can assume that $\psi_{1}, \psi_{2}$ are real spinors of length one and orthogonal at any point. The dimension of the spinor bundle $S$ is related to the dimension of the tangent bundle $T$ by

$$
\operatorname{dim} S=\operatorname{dim} T+1 \text {. }
$$

Consequently there exists a unique vector field $\xi$ such that

$$
\xi \psi_{1}=\psi_{2} .
$$

Moreover, we introduce the 1-form

$$
\eta(X):=\left(X \psi_{1}, \psi_{2}\right)
$$

as well as the endomorphism $\varphi: T \rightarrow T$

$$
\varphi(X):=-\nabla_{X} \xi
$$

Theorem $4\left(M^{7}, \varphi, \xi, \eta, g\right)$ is an Einstein-Sasakian manifold.

Proof. First we prove that $\xi$ is a Killing vector field, i.e.

$$
g\left(\nabla_{Y} \xi, X\right)+g\left(Y, \nabla_{X} \xi\right)=0 .
$$

We differentiate the equation $\xi \psi_{1}=\psi_{2}$ with respect to $Y$ and multiply it by $X \psi_{1}$ :

$$
\left(\left(\nabla_{Y} \xi\right) \psi_{1}, X \psi_{1}\right)+\frac{1}{2}\left(\xi Y \psi_{1}, X \psi_{1}\right)=\frac{1}{2}\left(Y \psi_{2}, X \psi_{1}\right)
$$

Analogously we have

$$
\left(\left(\nabla_{X} \xi\right) \psi_{1}, Y \psi_{1}\right)+\frac{1}{2}\left(\xi X \psi_{1}, Y \psi_{1}\right)=\frac{1}{2}\left(X \psi_{2}, Y \psi_{1}\right)
$$

Adding the equations $\langle 2\rangle$ and $\langle 3\rangle$ and taking into account

$$
(X \psi, Y \psi)=g(X, Y)|\psi|^{2}
$$

we obtain $\langle 1\rangle$. Therefore $\xi$ is a Killing vector field and the $(1,1)$-tensor $\varphi(X):=-\nabla_{X} \xi$ is antisymmetric. In particular we have $\eta(\xi)=1, \varphi(\xi)=0$ and

$$
\eta(X)=\left(X \psi_{1}, \psi_{2}\right)=\left(X \psi_{1}, \xi \psi_{1}\right)=g(X, \xi) .
$$

Now we verify the condition $\varphi^{2}=-\mathrm{Id}+\eta \otimes \xi$. Suppose that $X$ is orthogonal to $\xi-$ the remaining case is obvious. Then we have

$$
\begin{gathered}
\xi \psi_{1}=\psi_{2}, \\
\left(\nabla_{X} \xi\right) \psi_{1}+\frac{1}{2} \xi X \psi_{1}=\frac{1}{2} X \psi_{2} \\
\varphi(X) \psi_{1}=\xi X \psi_{1}
\end{gathered}
$$


and, consequently,

$$
\varphi^{2}(X) \psi_{1}=\xi \varphi(X) \psi_{1}=\xi \xi X \psi_{1}=-X \psi_{1},
$$

i.e.

$$
\varphi^{2}(X)=-X
$$

Moreover, we obtain

$$
\begin{aligned}
g(\varphi(X), \varphi(Y)) & =-g\left(\varphi^{2}(X), Y\right)=g(X-\eta(X) \xi, Y) \\
& =g(X, Y)-\eta(X) \eta(Y) .
\end{aligned}
$$

Since $\eta(X)=g(X, \xi)$ and taking into account that $\xi$ is a Killing vector field, we have

$$
d \eta(X, Y)=-2 g\left(X, \nabla_{Y} \xi\right)=2 g(X, \varphi(Y)) .
$$

Using a local frame in the reduction $Q\left(\psi_{1}, \psi_{2}\right)$ of the frame bundle we calculate

$$
\eta \wedge(d \eta)^{3}=48 d M^{7} \text {. }
$$

Finally it remains to prove the Sasakian integrability condition g) of Sect. 2. In the following calculations we apply the formula

$$
2 \varphi(Z) \psi_{1}=(\xi Z-Z \xi) \psi_{1}
$$

several times. We start with

$$
\nabla_{X}\left(\varphi(Y) \psi_{1}\right)=\nabla_{X}(\varphi(Y)) \psi_{1}+\frac{1}{2} \varphi(Y) X \psi_{1}
$$

and

$$
\begin{aligned}
\nabla_{X}\left(\varphi(Y) \psi_{1}\right)= & \nabla_{X}\left(-\frac{1}{2} Y \psi_{2}+\frac{1}{2} \xi Y \psi_{1}\right) \\
= & -\frac{1}{2}\left(\nabla_{X} Y\right) \psi_{2}-\frac{1}{4} Y X \psi_{2}-\frac{1}{2} \varphi(X) Y \psi_{1} \\
& +\frac{1}{2} \xi\left(\nabla_{X} Y\right) \psi_{1}+\frac{1}{4} \xi Y X \psi_{1} .
\end{aligned}
$$

Now we obtain:

$$
\begin{aligned}
\left(\nabla_{X} \varphi\right) & (Y) \psi_{1}=\left(\nabla_{X} \varphi(Y)\right) \psi_{1}-\varphi\left(\nabla_{X} Y\right) \psi_{1} \\
= & -\frac{1}{2}\left(\nabla_{X} Y\right) \psi_{2}-\frac{1}{4} Y X \psi_{2}-\frac{1}{2} \varphi(X) Y \psi_{1}+\frac{1}{2} \xi\left(\nabla_{X} Y\right) \psi_{1} \\
& +\frac{1}{4} \xi Y X \psi_{1}-\frac{1}{2} \varphi(Y) X \psi_{1}-\varphi\left(\nabla_{X} Y\right) \psi_{1} \\
= & -\frac{1}{4} Y X \psi_{2}-\frac{1}{2} \varphi(X) Y \psi_{1}+\frac{1}{4} \xi Y X \psi_{1}-\frac{1}{2} \varphi(Y) X \psi_{1} \\
= & -\frac{1}{4} Y X \psi_{2}+\frac{1}{2}(Y \varphi(X)+X \varphi(Y)) \psi_{1} \\
& -\frac{1}{4} Y \xi X \psi_{1}-\frac{1}{2} \eta(Y) X \psi_{1} \\
= & \frac{1}{2}(Y \varphi(X)+X \varphi(Y)) \psi_{1}+\frac{1}{2} \eta(X) Y \psi_{1}-\frac{1}{2} \eta(Y) X \psi_{1} \\
= & \frac{1}{4}(Y \xi X-Y X \xi+X \xi Y-X Y \xi) \psi_{1} \\
& +\frac{1}{2} \eta(X) Y \psi_{1}-\frac{1}{2} \eta(Y) X \psi_{1} \\
= & (g(X, Y) \xi-\eta(Y) X) \psi_{1} .
\end{aligned}
$$

This calculation yields

$$
\left(\nabla_{X} \varphi\right)(Y)=g(X, Y) \xi-\eta(Y) X,
$$

i.e. $\left(M^{7}, \varphi, \xi, \eta, g\right)$ is an Einstein-Sasakian manifold. 
Theorem 1 and Theorem 4 yield the following

Corollary. Let $M^{7}$ be a simply connected 7-dimensional spin manifold. Then there is a one-to-one correspondence between pairs of Killing spinors and Einstein-Sasakian structures on $M^{7}$.

Suppose now that the constructed Sasakian structure $(\varphi, \xi, \eta, g)$ on $M^{7}$ is regular, i.e. all integral curves of $\xi$ are closed and have the same length $L$ (see [5]). Then we have an $S^{1}$-action on $M^{7}$ and the orbit space is a 6-dimensional manifold $X^{6}$. Let us denote the projection from $M^{7}$ onto $X^{6}$ by $\pi$. We obtain a principal $S^{1}$-bundle $\pi: M^{7} \rightarrow X^{6}$ with the connection.

$$
\frac{2 \pi i}{L} \eta: T M^{7} \rightarrow \mathrm{Ri}=\underline{S}^{1} \text {. Since } \mathscr{L}_{\xi} g=0 \text { and } \mathscr{L}_{\xi} \varphi=0,
$$

$X^{6}$ admits a Riemannian metric and an almost complex structure which is integrable (see [5]). Denote by $\Omega$ the Kähler form of $X^{6}$. Then

$$
\pi^{*} \Omega(X, Y)=g(X, \varphi Y)=\frac{1}{2} d \eta(X, Y)
$$

and we conclude $d \Omega=0$, i.e. $X^{6}$ is a Kähler manifold. As $g$ is an Einstein metric on $M^{7}$, the O'Neill formulas yield that $X^{6}$ is a Kähler-Einstein manifold with the scalar curvature $\bar{R}=\frac{24}{21} R=48$. Next we study the topological type of the $S^{1}$-bundle $\pi: M^{7} \rightarrow X^{6}$. The curvature form of the connection $\frac{2 \pi i}{L} \eta$ is $\frac{2 \pi i}{L} d \eta$. Consequently the Chern class $c_{1}^{*} \in H^{2}(X, R)$ is given by $c_{1}^{*}=\left[\frac{1}{L} d \eta\right]$. On the other hand, since $X^{6}$ is a Kähler-Einstein manifold, its Chern class is given by the Ricci form

$$
c_{1}=\left[\frac{1}{2 \pi} \Omega_{\mathrm{Ric}}\right]=\left[\frac{1}{2 \pi} \frac{R}{6} \Omega\right]=\left[\frac{2}{\pi} d \eta\right] \text {, }
$$

and we obtain the relation $c_{1}=\frac{2 L}{\pi} c_{1}^{*}$ between the Chern class $c_{1}$ of $X^{6}$ and the Chern class $c_{1}^{*}$ of the $S^{1}$-bundle $\pi: M^{7} \rightarrow X^{6} . X^{6}$ is simply connected by the Kobayashi theorem (see [25]). We conclude from the Thom-Gysin sequence of the fibration $M^{7} \rightarrow X^{6}$ that

$$
H_{1}\left(M^{7} ; Z\right)=H^{6}\left(M^{7} ; Z\right)=H^{6}\left(X^{6} ; Z\right) / c_{1}^{*} \cup H^{4}\left(X^{6} ; Z\right) .
$$

Since the Killing spinors $\psi_{1}$ and $\psi_{2}$ on $M^{7}$ define a $S U(3)$-reduction $Q\left(\psi_{1}, \psi_{2}\right)$ of the frame bundle, we have an isomorphism

$$
\pi^{*} T_{\mathbb{C}} X^{6}=T^{h}=Q\left(\psi_{1}, \psi_{2}\right) \times_{s U(3)} \mathbb{C}^{3}
$$

of 3-dimensional complex vector bundles. Consequently we have

$$
\pi^{*} c_{1}\left(X^{6}\right)=0
$$

because the first Chern class of any $S U(3)$-bundle vanishes. Then the Thom-Gysin sequence yields

$$
c_{1} / c_{1}^{*} \in Z .
$$

Finally, from the exact homotopy sequence of the fibration $S^{1} \rightarrow M^{7} \rightarrow X^{6}$ we obtain that $\pi_{1}\left(M^{7}\right)$ is a cyclic group. 
This shows that in dimension 7 our general construction principle of Riemannian manifolds with two Killing spinors (Theorem 1 and Example 1) covers all solutions under the regularity assumption mentioned above, i.e.

Theorem 5. Let $\left(M^{7}, g\right)$ be a compact Riemannian spin manifold with two Killing spinors such that the induced contact structure is regular. Then $M^{7}$ is an $S^{1}$-bundle over a Kähler-Einstein manifold $X^{6}$ with positive scalar curvature and $c_{1}\left(N^{6}\right) / c_{1}^{*}$ is an integral cohomology class.

\section{The Classification of 7-Dimensional Manifolds with Three Independent Killing Spinors}

We consider a compact 7-dimensional Einstein manifold $\left(M^{7}, g\right)$ of scalar curvature $R=42$ admitting three orthogonal real Killing spinors $\psi_{1}, \psi_{2}, \psi_{3}$ of length one, i.e.

$$
\nabla_{X} \psi_{\alpha}=\frac{1}{2} X \psi_{\alpha}, \quad \alpha=1,2,3 .
$$

Solving the algebraic equations

$$
X_{1} \psi_{1}=\psi_{2}, \quad X_{2} \psi_{1}=\psi_{3}, \quad X_{3} \psi_{2}=\psi_{3}
$$

we obtain obviously three orthogonal Killing vector fields $X_{1}, X_{2}, X_{3}$ of length one with

$$
\left[X_{1}, X_{2}\right]=2 X_{3}, \quad\left[X_{2}, X_{3}\right]=2 X_{1}, \quad\left[X_{3}, X_{1}\right]=2 X_{2} .
$$

So $X_{1}, X_{2}, X_{3}$ induce a locally free isometric action of the group Spin(3) on $M^{7}$. We prove for example $\left[X_{1}, X_{2}\right]=2 X_{3}$. Since the $X_{\alpha}(1 \leqq \alpha \leqq 3)$ are Killing vector fields of length one, we have $g\left(\left[X_{1}, X_{2}\right], X_{1}\right)=0$. We differentiate the equations $X_{1} \psi_{1}$ $=\psi_{2}, X_{2} \psi_{1}=\psi_{3}$ with respect to $X_{2}$ and $X_{1}$ :

$$
\begin{aligned}
& \left(\nabla_{X_{2}} X_{1}\right) \psi_{1}+\frac{1}{2} X_{1} X_{2} \psi_{1}=\frac{1}{2} X_{2} \psi_{2}, \\
& \left(\nabla_{X_{1}} X_{2}\right) \psi_{1}+\frac{1}{2} X_{2} X_{1} \psi_{1}=\frac{1}{2} X_{1} \psi_{3} .
\end{aligned}
$$

Using the formula $\left[X_{1}, X_{2}\right]=\nabla_{X_{1}} X_{2}-\nabla_{X_{2}} X_{1}$ we obtain

$$
\left[X_{1}, X_{2}\right] \psi_{1}=2 X_{1} \psi_{3} \text {. }
$$

Now we multiply the last equation by $X_{1}$ and apply the orthogonality of $X_{1}$ to $\left[X_{1}, X_{2}\right]$

$$
\begin{gathered}
-\left[X_{1}, X_{2}\right] X_{1} \psi_{1}=-2 \psi_{3}, \\
-\left[X_{1}, X_{2}\right] \psi_{2}=-2 \psi_{3}=-2 X_{3} \psi_{2} .
\end{gathered}
$$

Let us recall the notion of a Sasakian 3-structure. Three Sasakian structures $\left(\varphi_{\alpha}, \xi_{\alpha}, \eta_{\alpha}\right)$ on a Riemannian manifold $\left(M^{n}, g\right)$ related by the conditions

$$
\begin{array}{cc}
\varphi_{3} \varphi_{2}=-\varphi_{1}+\eta_{2} \otimes \xi_{3}, & \varphi_{2} \varphi_{3}=\varphi_{1}+\eta_{3} \otimes \xi_{2}, \\
\varphi_{1} \varphi_{3}=-\varphi_{2}+\eta_{3} \otimes \xi_{1}, & \varphi_{3} \varphi_{1}=\varphi_{2}+\eta_{1} \otimes \xi_{3}, \\
\varphi_{2} \varphi_{1}=-\varphi_{3}+\eta_{1} \otimes \xi_{2}, & \varphi_{1} \varphi_{2}=\varphi_{3}+\eta_{2} \otimes \xi_{1}, \\
{\left[\xi_{1}, \xi_{2}\right]=2 \xi_{3}\left[\xi_{2}, \xi_{3}\right]=2 \xi_{1}\left[\xi_{3}, \xi_{1}\right]=2 \xi_{2}}
\end{array}
$$

constitute a Sasakian 3-structure. 
We remark that a Riemannian manifold with a Sasakian 3-structure is automatically an Einstein space of scalar curvature $R=n(n-1)$ (see [23]). Furthermore, let two Sasakian structures $\left(\varphi_{\alpha}, \xi_{\alpha}, \eta_{\alpha}\right), \alpha=1,2$, be given on a Riemannian manifold $\left(M^{2 k+1}, g\right)$ with orthogonal vector fields $\xi_{1}, \xi_{2}$. Then we have

$$
g\left(\nabla_{\xi_{1}} \xi_{2}, Z\right)=-g\left(\nabla_{Z} \xi_{2}, \xi_{1}\right)=g\left(\xi_{2}, \nabla_{Z} \xi_{1}\right)=-g\left(Z, \nabla_{\xi_{2}} \xi_{1}\right),
$$

and consequently

$$
\nabla_{\xi_{1}} \xi_{2}=-\nabla_{\xi_{2}} \xi_{1}
$$

Define now the vector field

$$
\xi_{3}:=\nabla_{\xi_{1}} \xi_{2}=-\nabla_{\xi_{2}} \xi_{1}=\varphi_{1}\left(\xi_{2}\right)=-\varphi_{2}\left(\xi_{1}\right)
$$

as well as the 1 -form $\eta_{3}$ and the $(1,1)$-tensor $\varphi_{3}$ by the formula

$$
\eta_{3}(X)=g\left(X, \xi_{3}\right), \quad \varphi_{3}(X)=-\nabla_{X} \xi_{3} .
$$

It seems to be well known and is not hard to prove that, in this situation, $\left(\varphi_{\beta}, \xi_{\beta}, \eta_{\beta}\right)$, $\beta=1,2,3$, is a Sasakian 3-structure, i.e. two Sasakian structures on $\left(M^{2 k+1}, g\right)$ with orthogonal Killing fields define a Sasakian 3-structure in a unique way. Finally we remark that for a given Sasakian 3-structure $\left(\varphi_{\beta}, \xi_{\beta}, \eta_{\beta}\right), \beta=1,2,3$, the corresponding volume forms $\eta_{\beta} \wedge\left(d \eta_{\beta}\right)^{k}$ induce the same orientation of $M^{2 k+1}$. Theorem 1 and Theorem 4 yield now

Theorem 6. Let $\left(M^{7}, g\right)$ be a compact Riemannian spin manifold with three independent Killing spinors. Then $\left(M^{7}, g\right)$ admits a Sasakian 3-structure. Conversely, every simply-connected spin manifold with Sasakian 3-structure admits at least three independent Killing spinors.

Proof. Three Killing spinors define Killing vector fields $X_{1}, X_{2}, X_{3}$ and, hence, three Sasakian structures $\eta_{\alpha}(X)=g\left(X, X_{\alpha}\right), \varphi_{\alpha}=-\nabla X_{\alpha}$ (Theorem 4). One can prove the formula relating $\varphi_{\alpha} \circ \varphi_{\beta}$ with $\varphi_{\gamma}$ in the same way as we derived the commutator relations between the vector fields $X_{\alpha}$ above. Conversely, given at least two Sasakian structures $\left(\varphi_{\alpha}, \xi_{\alpha}, \eta_{\alpha}\right), \alpha=1,2$, on a Riemannian manifold $\left(M^{7}, g\right)$ such that $\xi_{1}$ and $\xi_{2}$ are orthogonal, we consider the bundles

$$
E_{\alpha}^{ \pm}=\left\{\psi \in S:\left( \pm \varphi_{\alpha}(X)+\xi_{\alpha} X-X \xi_{\alpha}\right) \psi=0\right\} .
$$

Then $E_{1}^{+}$as well as $E_{2}^{+}$, or $E_{1}^{-}$as well as $E_{2}^{-}$have the dimension 2. Assume for example the first case. If $E_{1}^{+} \cap E_{2}^{+}=\{0\}$, then there exist at least four Killing spinors on $M$ according to Theorem 1, and consequently $M^{7}$ is isometric to the sphere $S^{7}$ (Theorem 3). Suppose $E_{1}^{+} \cap E_{2}^{+} \neq\{0\}$ and fix a spinor $\psi \neq 0$ in $E_{1}^{+} \cap E_{2}^{+}$. Then, by the definition of $E_{\alpha}^{+}$the spinors $\xi_{1} \psi$ and $\xi_{2} \psi$ are elements of $E_{1}^{+}$and $E_{2}^{+}$, respectively, i.e. $\psi, \xi_{1} \psi$, and $\xi_{2} \psi$ are elements of $E_{1}^{+}+E_{2}^{+}$. However, because of $\left(\xi_{1} \psi, \xi_{2} \psi\right)=g\left(\xi_{1}, \xi_{2}\right)|\psi|^{2}=0$, these three spinors are orthogonal. Hence $E_{1}^{+} \neq E_{2}^{+}$ and consequently $M^{7}$ admits at least 3 Killing spinors (Theorem 1).

Now we want to classify all 7-dimensional compact Riemannian manifolds with three Killing spinors under a certain regularity assumption about the Spin(3)action on $M^{7}$. We use the technique and notation of twistor theory, associating to a conformal 4-dimensional manifold $X^{4}$ an (almost)-complex 3-dimensional manifold $Z^{-}\left(X^{4}\right)$ as well as a fibration $q: Z^{-}\left(X^{4}\right) \rightarrow X^{4}$. General references for this are the papers $[1,16,34]$. In particular we use the classification of all compact Kähler 
twistor spaces obtained in $[15,21]$ in our classification. We suppose that $M^{7}$ is a simply-connected compact spin manifold with three Killing spinors and $M^{7} / \operatorname{Spin}(3)=: X^{4}$ is a smooth closed manifold. In this case $M^{7}$ is an $S^{1}$-fibration over the twistor space $Z^{-}$of $X^{4}$. Indeed, let $p: M^{7} \rightarrow X^{4}$ be the projection and identify the tangent space $T_{p(m)} X^{4}$ with the orthogonal complement of $\operatorname{Lin}\left(X_{1}, X_{2}, X_{3}\right)$ in $T_{m} M^{7}$. We define the projection $\pi: M^{7} \rightarrow Z^{-}$by the formula

$$
\pi(m)=d p \varphi_{1}(m) d p^{-1} .
$$

Then the kernel of the differential $d \pi: T_{m} M^{7} \rightarrow T_{\pi(m)} Z^{-}$is generated by $X_{1}$ and consequently $\pi: M^{7} \rightarrow Z^{-}$is an $S^{1}$-fibration. This projection coincides with the corresponding projection for two Killing spinors $\psi_{1}, \psi_{2}$ considered in Sect. 5. Theorem 5 provides now.

Lemma 1. $\pi: M^{7} \rightarrow Z^{-}$is an $S^{1}$-fibration and $Z^{-}$is a compact Kähler-Einstein manifold of scalar curvature $\bar{R}=48$. The canonical complex structure of $Z^{-}$is given by $\varphi_{1}$.

Since the only Kählerian twistor spaces are $P^{3}(\mathbb{C})$ and the flag manifold $F(1,2)$ (see $[15,21]), Z^{-}$is analytically equivalent to one of these spaces. Moreover, on $P^{3}(\mathbb{C})$ and $F(1,2)$ there exists only one Kähler-Einstein structure (see [24]) and consequently $Z^{-}$is analytically isometric to $P^{3}(\mathbb{C})$ or $F(1,2)$. Now we carefully investigate the action of $\operatorname{Spin}(3)$ on $M^{7}$. For a given point $m \in M^{7}$ we denote by

$$
H(m)=\{\gamma \in \operatorname{Spin}(3): \gamma m=m\}
$$

the isotropy group of this point.

Lemma 2. For any point $m \in M^{7}$ the isotropy group $H(m)$ is trivial or is isomorphic to $Z_{2}$.

Proof. Denote by $H$ the subgroup of Spin(3) given by the vector field $X_{1}$. Consider a point $m \in M^{7}$ as well as $x=p(m) \in X^{4}$ and the fibres $M_{x}^{7}=p^{-1}(x), Z_{x}^{-}=q^{-1}(x)$. Then the diagram

$$
\begin{aligned}
& \operatorname{Spin}(3) \rightarrow \operatorname{Spin}(3) / H(m)=M_{x}^{7} \stackrel{\pi}{\longrightarrow} Z_{x}^{-}=H \backslash(\operatorname{Spin}(3) / H(m)) \\
& \Downarrow \\
& H \backslash \operatorname{Spin}(3) \longrightarrow(H \backslash \operatorname{Spin}(3)) / H(m)
\end{aligned}
$$

commutes and therefore the map $H \backslash \operatorname{Spin}(3) \rightarrow(H \backslash \operatorname{Spin}(3)) / H(m)$ is a covering. With respect to $(H \backslash \operatorname{Spin}(3)) / H(m)=Z_{x}^{-}=S^{2}$ the mentioned map is one-to-one, i.e. $H(m)$ acts trivially on $H \backslash \operatorname{Spin}(3)$. Now we identify $H \backslash \operatorname{Spin}(3)$ with $S^{2}=\mathbb{C} \cup\{\infty\}$ and see that the corresponding action of $\operatorname{Spin}(3) \approx S U(2)$ is given by

$$
z \rightarrow \frac{A z+B}{C z+D}\left(\begin{array}{ll}
A & B \\
C & D
\end{array}\right) \in S U(2) .
$$

Since $H(m) \subset S U(2)$ acts trivially on $\mathbb{C} \cup\{\infty\}$, we obtain $H(m)=\{e\}$ or $Z_{2}$.

Lemma 3. The orbit type of the Spin(3)-action on $M^{7}$ is constant, i.e. there are two possible cases: either $H(m)=\{e\}$ or $H(m)=Z_{2}$ for all points $m \in M^{7}$.

Proof. Consider $\gamma=(-1) \in \operatorname{Spin}(3)$ and the corresponding isometric involution $\gamma=(-1): M^{7} \rightarrow M^{7}$. The fixed point set of $\gamma$ is the union of closed totally geodesic 
submanifolds $N_{\alpha}$. The manifolds $N_{\alpha}$ are Spin(3)-invariant and $S O(3)$ $=\operatorname{Spin}(3) /\{ \pm 1\}$ acts freely on it.

Furthermore, the dimension of each $N_{\alpha}$ equals 3,5 or 7 . If at least one of the submanifolds $N_{\alpha}$ has dimension 7, it follows that $N_{\alpha}=M^{7}$ and, consequently, $H(m)=Z_{2}$ for all points $m \in M^{7}$. Next we show that the case $\operatorname{dim} N_{\alpha}=5$ is impossible. Indeed, if the fixed point set of $\gamma$ has a component $N_{\alpha}$ of dimension 5, we consider the images of $N_{\alpha}$ in $X^{4}$ and $Z^{-}$. Then $\Sigma^{2}=p\left(N_{\alpha}\right) \subset X^{4}$ is a surface and $\pi\left(N_{\alpha}\right) \subset Z^{-}$is a complex submanifold of the twistor space $Z^{-}$. In fact, the tangent space of $N_{\alpha}$ contains all vectors $t$ invariant under the differential $d \gamma$ of the isometry $\gamma$. Then

$$
d \gamma \varphi_{1}(\mathbf{t})=-d \gamma \nabla_{\mathbf{t}} X_{1}=-\nabla_{d \gamma(\mathbf{t})} d \gamma\left(X_{1}\right)=-\nabla_{\mathbf{t}} X_{1}=\varphi_{1}(\mathbf{t}),
$$

i.e. $T N_{\alpha}$ is $\varphi_{1}$-invariant. Now we regard the twistor projection $q: Z^{-} \rightarrow X^{4}$. Then we have $q^{-1}\left(\Sigma^{2}\right)=\pi\left(N_{\alpha}\right)$.

Since $\pi\left(N_{\alpha}\right)$ is a complex submanifold, the last equation means, by definition of the twistor space, that $T_{x} \Sigma^{2}$ is invariant under all algebraic complex structures of $T_{x} X^{4}$, a contradiction.

Finally we prove that the fixed point set of $\gamma$ cannot contain a component of dimension 3. Suppose $\operatorname{dim} N_{\alpha}=3$ and take a tubular neighbourhood $U=\operatorname{Spin}(3) \times{ }_{z_{2}} D^{4}$. Then we have

$$
\pi_{1}(U)=\pi_{1}\left(N_{\alpha}\right)=\pi_{1}(\operatorname{Spin}(3) /\{ \pm 1\})=Z_{2} \quad \text { and } \quad \pi_{1}\left(U \backslash N_{\alpha}\right)=0,
$$

since $U \backslash N_{\alpha}$ is diffeomorphic to $\operatorname{Spin}(3) \times\left(D^{4} \backslash\{0\}\right)$. The van Kampen theorem implies now that $\pi_{1}\left(M^{7}\right)$ contains a subgroup of order two, a contradiction to the assumption $\pi_{1}\left(M^{7}\right)=0$.

We explain now the classification of simply-connected Riemannian manifolds with three Killing spinors. First of all we remark that one knows two such spaces, namely the 7-dimensional sphere $S^{7}$ and the space $S U(3) / S_{1,1}^{1}=N(1,1)$ described in [6]. We prove that, under the regularity assumption on the Spin(3)-action, these are the only possible spaces with three Killing spinors.

Theorem 7. Let $M^{7}$ be a compact simply connected Riemannian spin manifold with three Killing spinors such that $M^{7} / \mathrm{Spin}(3)$ becomes a smooth closed manifold for the induced Spin(3)-action. Then $M^{7}$ is isometric to the sphere $S^{7}$ or the space $S U(3) / S_{1,1}^{1}$ $=N(1,1)$.

Proof. We consider the map $\pi: M^{7} \rightarrow Z^{-}$. Since the isotropy group $H(m)$ is constant, this map is the projection of a principal $S^{1}$-fibre bundle. On the other hand, $Z^{-}$is a Kähler-Einstein twistor space and therefore isometric to the complex projective space $P^{3}(\mathbb{C})$ or to the flag manifold $F(1,2)([15,21,24])$. In case $Z^{-}=P^{3}(\mathbb{C})$, we have $c_{1}\left(P^{3}(\mathbb{C})\right)=4 \alpha$, where $\alpha \in H^{2}\left(P^{3}(\mathbb{C}) ; Z\right)$ is the generator of the second cohomology group. Since $\pi_{1}\left(M^{7}\right)=0$, the Chern class of the fibration $\pi: M^{7}$ $\rightarrow P^{3}(\mathbb{C})$ must be equal to $c_{1}^{*}=\alpha$, and with respect to the relation $c_{1}=\frac{2 L}{\pi} c_{1}^{*}$ we obtain $L=2 \pi$ for the length $L$ of the circles of this fibration (see Sect. 5). These data determine $M^{7}$ up to an isometry and it turns out that $M^{7}$ is isometric to the sphere $S^{7}$.

We handle the second case similarly. Then we have $Z^{-}=F(1,2)$. The group $H^{2}(F(1,2) ; Z)$ has two generators $\alpha, \gamma$ and the first Chern class is given by $c_{1}(F(1,2))$ $=2 \gamma$. Therefore the Chern class $c_{1}^{*}$ of the fibration $\pi: M^{7} \rightarrow F(1,2)$ and the length $L$ 
are given by $c_{1}^{*}=\gamma$ and $L=\pi$. Again, these data describe $M^{7}$ uniquely and it becomes isometric to $S U(3) / S_{1,1}^{1}=N(1,1)$.

Corollary. Every compact simply connected 7-dimensional spin manifold with regular Sasakian 3-structure is isometric to $S^{7}$ or $S U(3) / S_{1,1}^{1}=N(1,1)$.

\section{Parallel Spinors on 7-Dimensional Manifolds}

The method used in the classification of 7-dimensional manifolds with Killing spinors can be applied to manifolds with parallel spinors, too. In particular we show

Theorem 8. Let $M^{7}$ be a non-flat compact 7-dimensional spin manifold admitting at least three parallel spinors. Then there exist a K3-surface $F$ with an anti-selfdual Ricci-flat Riemannian metric, a lattice $\Gamma \subset R^{3}$ and a representation $\varrho: \Gamma \rightarrow \operatorname{Aut}_{h^{2}}(\Gamma)$ of $\Gamma$ in the group of all automorphisms of the $K 3$ surface $F$ preserving the unique holomorphic two-form $h^{2}$ such that $M^{7}$ is isometric to

$$
M^{7}=R^{3} \times F \mid \Gamma,
$$

where $\Gamma$ acts on $R^{3} \times F$ by $\gamma \cdot(x, f)=(x+\gamma, \varrho(\gamma) f)$. Conversely, any 7-dimensional Riemannian manifold of this type admits at least four parallel spinors.

Proof. Let $\psi_{1}, \psi_{2}, \psi_{3}$ be three orthogonal parallel spinors of length 1. In the same way as in Sect. 6, $\psi_{1}, \psi_{2}, \psi_{3}$ define the vector fields $X_{1}, X_{2}, X_{3}$. These vector fields as well as the 1 -forms $\eta_{\alpha}=g\left(X_{\alpha}, \cdot\right)$ are parallel. So we have, by the Frobenius theorem, a foliation $M^{7}=\bigcup F_{\alpha}^{4}$ of $M^{7}$ into totally geodesic, connected, complete manifolds $F_{\alpha}^{4}$. Furthermore, the leaves $F_{\alpha}^{4}$ are antiselfdual and Ricci-flat. This follows immediately from the structure of the Lie algebra $h\left(u_{1}, u_{2}, u_{3}\right)$ described in Proposition 1. We have $b_{1}\left(M^{7}\right) \leqq 3$ (see [7]). On the other hand, the 1-forms $\eta_{1}, \eta_{2}, \eta_{3}$ are linearly independent, and consequently $b_{1}\left(M^{7}\right)=3$. We fix a basis $\alpha_{1}, \alpha_{2}, \alpha_{3}$ in the torsion-free part of $H_{1}\left(M^{7} ; Z\right)$ and consider the homomorphism $L: \pi_{1}\left(M^{7}\right) \rightarrow R^{3}$ given by

$$
L(\gamma)=\left(\int_{\gamma} \eta_{1}, \int_{\gamma} \eta_{2}, \int_{\gamma} \eta_{3}\right)
$$

The vectors $L\left(\alpha_{1}\right), L\left(\alpha_{2}\right), L\left(\alpha_{3}\right)$ are linearly independent in $R^{3}$ because

$$
A_{1} L\left(\alpha_{1}+A_{2} L\left(\alpha_{2}\right)+A_{3} L\left(\alpha_{3}\right)=0\right.
$$

implies $\int_{A_{1} \alpha_{1}+A_{2} \alpha_{2}+A_{3} \alpha_{3}} \eta_{i}=0(i=1,2,3)$, and therefore $A_{1} \alpha_{1}+A_{2} \alpha_{2}+A_{3} \alpha_{3}=0$ in $H_{1}\left(M^{7} ; R\right)$. Let $\Gamma$ be a lattice generated by $L\left(\alpha_{1}\right), L\left(\alpha_{2}\right), L\left(\alpha_{3}\right)$. Then we obtain a submersion $f: M^{7} \rightarrow R^{3} / \Gamma$ defined by

$$
f(m)=\left(\int_{c} \eta_{1}, \int_{c} \eta_{2}, \int_{c} \eta_{3}\right) \bmod \Gamma
$$

where $c$ is a curve from a fixed point $m_{0}$ to $m$.

Since $T_{x} F_{\alpha}^{4}=\left\{t \in T_{x} M^{7}: d f(t)=0\right\}$, the leaves of the foliation $U F_{\alpha}^{4}$ are contained in the fibres of the submersion $f$. In case the fibres $f^{-1}(x)$ are not connected, there exist a covering of the torus $T^{3}=R^{3} / \Gamma$ and a lift of $f$ into this space. Therefore we may assume that the fibres of $f$ are connected and coincide with the leaves $F_{\alpha}^{4}$. 
Since the normal bundle of any fibre is trivial, any fibre is a compact connected anti-self-dual Ricci-flat Riemannian spin manifold, i.e. either a flat space or a $K 3$-surface (see [20]). If at least one fibre is flat, then all fibres are flat and, consequently, $M^{7}$ is a flat Riemannian manifold. Therefore we may assume that $M^{7}$ is a fibration over $T^{3}=R^{3} / \Gamma$ with totally geodesic fibres isometric to a $K 3$-surface $F$. Consider the covering $R^{3} \rightarrow R^{3} / \Gamma$ as well as the induced fibration $\tilde{M}^{7} \rightarrow R^{3}$ over $R^{3}$. Then $M^{7}$ is isometric to $\tilde{M}^{7} / \Gamma$. On the other hand, since the parallel transport in a Riemannian submersion with totally geodesic fibres maps any fibre isometrically on any other fibre, it turns out that $\tilde{M}^{7}$ is isometric to $R^{3} \times F$. The action of $\Gamma$ on $F$ preserves the holomorphic structure as well as the unique holomorphic 2-form $h^{2}$. Indeed, the holomorphic structure of any $K 3$-surface is given by the parallel spinors on it and $h^{2}$ is one of the two parallel spinors under the isomorphism $S=\Lambda^{0,0} \oplus \Lambda^{0,2}$ of the spin bundle $S$. We restrict the parallel spinors $\psi_{1}, \psi_{2}, \psi_{3}$ on $M^{7}$ to the fibre $F$. Since the restriction of the sevendimensional spin representation to the subgroup Spin(4) is equivalent to $\Delta_{4} \oplus \Delta_{4}$, each $\left.\psi_{i}\right|_{F}(i=1,2,3)$ corresponds to a pair of parallel spinors on $F$. The $\Gamma$-action on $\Gamma$ preserves $\left.\psi_{i}\right|_{F}$ since the $\psi_{i}$ are parallel on $M^{7}$. Consequently $\Gamma$ acts on $F$ holomorphically and preserves $h^{2}$.

Theorem 9. Let $M^{7}$ be a compact non-flat Riemannian manifold with two parallel spinors. Then either $M^{7}$ admits at least four parallel spinors and is isometric to $R^{3} \times F / \Gamma$ for a certain K3-surface $F$ or there exists a Ricci-flat compact Kähler manifold $N^{6}$ and a holomorphic isometry $\Phi: N^{6} \rightarrow N^{6}$ such that $M^{7}$ is isometric to $M^{7}=N^{6} \times[0,1] / \sim$ with the identification $(x, 0) \sim(\phi(x), 1)$.

Proof. Consider two parallel spinors $\psi_{1}, \psi_{2}$ as well as the parallel 1-form $\eta$ defined by $\eta \psi_{1}=\psi_{2} . M^{7}$ is a compact Ricci-flat Riemannian manifold (see [10]) and the first Betti number $b_{1}\left(M^{7}\right)$ is at least one. In case $b_{1}\left(M^{7}\right)=1$, we can prove (see [13]) that the leaves of the foliation given by $\eta$ are fibres of a Riemannian submersion $f: M^{7} \rightarrow S^{1}$ with totally geodesic Ricci-flat and Kählerian fibres $N^{6}$. Using the parallel transport defined by the vector field corresponding to $\eta, M^{7}$ becomes isometric to $N^{6} \times[0,1] / \sim$ for some $\Phi: N^{6} \rightarrow N^{6}$. $\Phi$ preserves $\left.\psi_{1}\right|_{N^{6}}$ and $\left.\psi_{2}\right|_{N^{6}}$. Consequently $\Phi$ is holomorphic. If $b_{1}\left(M^{7}\right) \geqq 2$, there exists a harmonic 1-form $\eta_{1}$ orthogonal to $\eta$ in $L^{2}$. The Weitzenböck formula $0=\Delta \eta_{1}=\nabla^{*} \nabla \eta_{1}+\operatorname{Ric}\left(\eta_{1}\right)$ yields that $\eta_{1}$ is a parallel 1 -form orthogonal to $\eta$ at any point of $M$. Then $\psi_{1}, \eta \psi_{1}=\psi_{2}$, and $\eta_{1} \cdot \psi_{1}:=\psi_{3}$ are orthogonal and parallel spinors on $M^{7}$.

\section{References}

1. Atiyah, M.F., Hitchin, N., Singer, I.M.: Self-duality in four-dimensional Riemannian geometry. Proc. R. Soc. Lond. Ser. A 326, 425-461 (1978)

2. Baum, H.: Odd-dimensional Riemannian manifolds with imaginary Killing spinors. Ann. Global Anal. Geom. 7, 2 (1989)

3. Baum, H.: Complete Riemannian manifolds with imaginary Killing spinors. Ann. Global Anal. Geom. (to appear)

4. Berger, M., Gauduchon, P., Mazet, E.: Le spectre d'une variété Riemannienne. Lecture Notes in Mathematics, Vol. 194. Berlin, Heidelberg, New York: Springer 1971

5. Blair, D.: Contact manifolds in Riemannian geometry. Lecture Notes in Mathematics, Vol. 509. Berlin, Heidelberg, New York: Springer 1976

6. Duff, M.J., Nilsson, B., Pope, C.N.: Kaluza-Klein supergravity. Phys. Rep. 130, 1-142 (1986)

7. Fischer, A.E., Wolf, J.A.: The structure of compact Ricci-flat Riemannian manifolds. J. Diff. Geom. 10, 277-288 (1975) 
8. Friedrich, Th.: Der erste Eigenwert des Dirac-Operators einer kompakten Riemannschen Mannigfaltigkeit nichtnegativer Skalarkrümmung. Math. Nachr. 97, 117-146 (1980)

9. Friedrich, Th.: A remark on the first eigenvalue of the Dirac operator on 4-dimensional manifolds. Math. Nachr. 102, 53-56 (1981)

10. Friedrich, Th.: Zur Existenz paralleler Spinorfelder über Riemannschen Mannigfaltigkeiten. Colloq. Math. 44, 277-290 (1981)

11. Friedrich, Th., Grunewald, R.: On the first eigenvalue of the Dirac operator on 6-dimensional manifolds. Ann. Global Anal. Geom. 3, 265-273 (1985)

12. Friedrich, Th., Kath, I.: Einstein manifolds of dimension five with small eigenvalue of the Dirac operator, J. Diff. Geom. 29, 263-279 (1989)

13. Friedrich, Th., Kath, I.: Compact 5-dimensional Riemannian manifolds with parallel spinors, Math. Nachr. (to appear)

14. Friedrich, Th., Kath, I.: Variétés Riemanniennes compactes de dimension 7 admettant des spineurs de Killing. C.R. Acad. Sci. Paris Ser. I. t. 307, 967-969 (1988)

15. Friedrich, Th., Kurke, H.: Compact four-dimensional self-dual Einstein manifolds with positive scalar curvature. Math. Nachr. 106, 271-299 (1982)

16. Friedrich, Th. (Ed.): Self-dual Riemannian geometry and instantons. Leipzig: Teubner 1981

17. Futaki, A.: Kähler-Einstein metrics and integral invariants. Lecture Notes in Mathematics, Vol. 1314. Berlin, Heidelberg, New York: Springer 1988

18. Hijazi, O.: Caractérisation de la sphère par les premières valeurs propres de l'opérateur de Dirac en dimension 3, 4, 7 et 8. C.R. Acad. Sci. Paris, Ser. I 303, 417-419 (1986)

19. Hijazi, O.: A conformal lower bound for the smallest eigenvalue of the Dirac operator and Killing spinors. Commun. Math. Phys. 104, 151-162 (1986)

20. Hitchin, N.: Compact four-dimensional Einstein manifolds. J. Diff. Geom. 9, 435-441 (1974)

21. Hitchin, N.: Kählerian twistor spaces. Proc. Lond. Math. Soc. 43, 133-150 (1981)

22. Husemoller, D.: Fibre bundles. New York: 1966

23. Ishihara, S., Konishi, M.: Differential geometry of fibred spaces. Kyoto: 1973

24. Kirchberg, K.D.: Compact six-dimensional Kähler spin manifolds of positive scalar curvature with the smallest positive first eigenvalue of the Dirac operator. Math. Ann. 282, 157-176 (1988)

25. Kobayashi, S.: On compact Kähler manifolds with positive definite Ricci tensor. Ann. Math. 74, 570-574 (1961)

26. Kobayashi, S.: Topology of positively pinched Kähler geometry. Tohoku Math. J. 15, 121-139 (1963)

27. Koiso, N., Sakane, Y.: Non-homogeneous Kähler-Einstein metrics on compact complex manifolds. Preprint

28. Kostant, B.: Quantization and unitary representations. Lecture Notes in Mathematics, Vol. 170, pp. 87-207. Berlin, Heidelberg, New York: Springer 1970

29. Kreck, M., Stolz, St.: A diffeomorphism classification of 7-dimensional homogeneous Einstein manifolds with $S U(3) \times S U(2) \times U(1)$-symmetry. Ann. Math. 127, 373-388 (1988)

30. Lichnerowicz, A.: Spin manifolds, Killing spinors, and universality of the Hijazi inequality. Lett. Math. Phys. 13, 331-344 (1987)

31. Lichnerowicz, A.: Les spineurs-twisteurs sur une varieté spinorielle compacte. C.R. Acad. Sci. Paris, Ser. I 306, 381-385 (1988)

32. Nieuwenhuizen, P. van, Warner, N.P.: Integrability conditions for Killing spinors. Commun. Math. Phys. 93, 277-284 (1984)

33. Postnikov, M.M.: Lectures on Geometry V, Lie groups and algebras. Moscow: 1982 (Russ.)

34. Salamon, S.: Topics in four-dimensional Riemannian geometry. In: Geometry Seminar "Luigi Bianchi". Lecture Notes in Mathematics, Vol. 1022. Berlin, Heidelberg, New York: Springer 1983

35. Tian, G.: On Kähler-Einstein metrics on certain Kähler manifolds with $c_{1}(M)>0$. Invent. Math. 89, 225-246 (1987)

36. Tian, G., Yau, S.-T.: Kähler-Einstein metrics on complex surfaces with $c_{1}>0$. Commun. Math. Phys. 112, 175-203 (1987)

37. Séminaire Palaiseau, Géométrie des surfaces K3: modules et périodes. Asterisque 126 (1985) 
